\title{
EDITORIAL
}

\section{Travelling wave ion mobility}

\section{Kevin Giles}

Published online: 19 April 2013

(C) Springer-Verlag Berlin Heidelberg 2013

The research papers in this issue of the International Journal for Ion Mobility Spectrometry are the concluding articles of a two-part special issue on Travelling Wave Ion Mobility. The first issue, published in March (Volume 16, Issue 1, March 2013), covered a wide range of research areas: 'Resolving the microcosmos of complex samples: UPLC/travelling wave ion mobility separation high resolution mass spectrometry for the analysis of in vivo drug metabolism studies' by Blech and Laux; 'The effects of cation adduction upon the conformation of three-helix bundle protein domains' by Sokratous et al.; 'Monitoring oligomer formation from self-aggregating amylin peptides using ESI-IMS-MS' by Young et al.; 'Traveling-wave ion mobility-mass spectrometry reveals additional mechanistic details in the stabilization of protein complex ions through tuned salt additives' by Han and Ruotolo; 'Coupling electrospray corona discharge, charge reduction and ion mobility mass spectrometry: From peptides to large macromolecular protein complexes' by Campuzano and Schnier; and 'Structural studies of metal ligand complexes by ion mobility-mass spectrometry' by Wright et al. The papers in this issue extend upon the range of studies presented in the first issue and together they illustrate the broad utility and applicability of (travelling wave) ion mobility - mass spectrometry.

Since the launch of the first travelling wave ion mobility system (SYNAPT) some 7 years ago, the interest in ion mobility coupled with mass spectrometry has grown tremendously and shows no sign of abating. We continue to push the boundaries of ion mobility instrumentation design and, together with our customers and collaborators, work to extend the applications of this technology. I am indebted to the small cross-section of our customers/collaborators who have taken the time to contribute to this special issue on Travelling Wave Ion Mobility and offer my sincere thanks.

\section{Kevin Giles}

Manchester 2013
K. Giles $(\bowtie)$

Waters Corporation, Floats Road,

Manchester M23 9LZ, UK

e-mail: Kevin_Giles@waters.com 